\title{
Limitations of cosmography in extended theories of gravity
}

\author{
Álvaro de la Cruz-Dombriz* \\ Astrophysics, Cosmology and Gravity Centre (ACGC), Department of Mathematics and Applied \\ Mathematics, University of Cape Town, Rondebosch 7701, Cape Town, South Africa \\ E-mail: alvaro.delacruzdombriz[at] uct.ac.za
}

\begin{abstract}
The cosmographic approach, which only relies upon the homogeneity and isotropy of the Universe on large scales, has become an essential tool in dealing with an increasing number of theoretical possibilities for explaining the late-time acceleration of the Universe, ranging from Modified Gravity theories to Dark Energy alternatives passing from testing the cosmological concordance $\Lambda \mathrm{CDM}$ model. Despite its generality, we show that this method has a number of shortcomings when trying to adequately reconstruct theories with higher-order derivatives in either the gravitational or the matter sector. Herein some paradigmatic examples of such an inability, explanations of the limitations and prospective cures will be presented.
\end{abstract}

The 11th International Workshop Dark Side of the Universe 2015

14-18 December 2015

Kyoto, Japan

${ }^{*}$ Speaker. 


\section{Overview}

Model-independent methods in cosmology have tried for years to make predictions on the correct underlying theory of gravity without making a priori assumptions on case-by-case classes of theories nor on specific models therein. Such methods, which rely on some basic symmetries of the spacetime structure under consideration, have intended to infer the dark energy equation of state, reconstruct classes of dark energy theories and find any hints which unveil departures from both the Einsteinian gravity and the Copernican principle (c.f. [1] for reviews). Among them, the assumption of the Copernican Principle (leading to the Friedmann-Lemaittre-Robertson-Walker (FLRW) metric) and the expression of the scale factor as a function of an auxiliary variable - for instance either time or redshifts - lead to the so-called cosmographic approach [2] which aims to reconstruct the underlying cosmological dynamics using comparison to data derived from such two basic assumptions. Cosmography has thus tried to spot deviations from the standard $\Lambda$ CDM model, to reconstruct models for dark energy and to even shed some light on the form of the gravitational Lagrangian for classes of modified (extended) gravity theories [3, 4, 5, 6, 7, 8].

Technicalities of Cosmography are widely known and we refer the reader to more detailed literature [9]. The most standard approach assumes as an auxiliary variable the usual redshift $z$ and then performs an expansion in the derivatives of the FLRW scale factor. Thus the Hubble parameter and its derivatives can be rewritten in terms of the well-known cosmographic parameters. One is then tempted to think that these parameters can be directly fitted with observational data, and that such a process leads to model (or theory)-independent constraints enabling reconstruction of the underlying cosmological models (or theories). As an alternative to the independent variable $z$, the above expansion may be expressed in terms of a variables ensuring the convergence of the series for the whole history of the Universe (c.f. $[4,10]$ for some possibilities). Nonetheless, the use of other redshifts, such as $y=z /(1+z)$ will be shown below to present strong limitations.

As a starting point, Cosmography was recently shown [11] to suffer from shortcomings even to target $\Lambda \mathrm{CDM}$ as the underlying theory when SNIa mock data [12] are precisely generated from a $\Lambda$ CDM model and studied by means of the cosmographic method when several expansion orders or auxiliary redshift are considered. In Table 1 as taken from [11] it is shown how the $y$ parametrisation gives completely biased estimators, the trends being as follows: the $y$-parametrisation provides much bigger errors, biasing $q_{0}$ to smaller values and $j_{0}$ and $s_{0}$ to greater values than the true ones. In fact only a few a extensive simulations lie within $1 \sigma$ whereas most of them do at $3 \sigma$ or more [11]. Moreover, the consideration of different number of parameters, namely $\theta_{1}$ and $\theta_{2}$ as described in Table 1, in the cosmographic expansion does not fix this limitation to correctly trace the exact values for the $\Lambda \mathrm{CDM}$ cosmographic parameters. In fact, as seen in Table 1 as taken from [11], the inclusion of one extra parameter (namely $l_{0}$ in $\theta_{2}$ ) leads to error overestimates for both $z$ and $y$ variables. Consequently a first caveat in the standard cosmographic approach lies precisely in which variable to use: analysis in [11] led to conclude that despite the fact that $y$ might seem a more appealing variable from a theoretical point of view, it clearly turns out to not even be appropriate to derive cosmological constraints in $\Lambda \mathrm{CDM}$, since fittings obtained in this variable are completely biased.

Another consistency check of Cosmography consists of testing the ability of the method to spot the Concordance $\Lambda \mathrm{CDM}$ model against other competitive theories with constant (although 
Table 1: Coverage test for two sets of parameters: $\theta_{\mathbf{1}}=\left\{H_{0}, q_{0}, j_{0}, s_{0}\right\}$ and $\theta_{\mathbf{2}}=\left\{H_{0}, q_{0}, j_{0}, s_{0}, l_{0}\right\}$. We refer the reader to [11] for further details.

\begin{tabular}{|c|c|c|c|c|c|c|c|c|c|c|c|c|}
\hline & & \multicolumn{5}{|c|}{$\theta_{1}$} & \multicolumn{6}{|c|}{$\theta_{2}$} \\
\hline & & $y$ & & & $z$ & & & $y$ & & & $z$ & \\
\hline & $1 \sigma$ & $2 \sigma$ & $3 \sigma$ & $1 \sigma$ & $2 \sigma$ & $3 \sigma$ & $1 \sigma$ & $2 \sigma$ & $3 \sigma$ & $1 \sigma$ & $2 \sigma$ & $3 \sigma$ \\
\hline$q_{0}$ & 26 & 32 & 42 & 67 & 27 & 6 & 82 & 12 & 6 & 82 & 18 & 0 \\
\hline$j_{0}$ & 10 & 45 & 45 & 64 & 29 & 7 & 93 & 5 & 2 & 88 & 12 & 0 \\
\hline$s_{0}$ & 10 & 67 & 23 & 83 & 15 & 2 & 92 & 7 & 1 & 93 & 6 & 1 \\
\hline$l_{0}$ & - & - & - & - & - & - & 100 & 0 & 0 & 100 & 0 & 0 \\
\hline
\end{tabular}

slightly different from $\omega=-1$ ) dark-energy equation of state, i.e., XCDM models. Once again, the use of mock SNIa data can serve to illustrate the limitations of the method; this time those mock data have been obtained from a flat XCDM model with cosmological parameters $\Omega_{m}=0.3$ and $\omega_{X}=-1.3$. In Fig. 1 we have considered two cosmographic realisations $\theta_{1}$ (fourth order) and $\theta_{2}$ (fifth order) in th $z$-redshift expansion, which have then been compared to the direct fitting of free parameters $\left\{\Omega_{m}, \omega_{X}\right\}$ in the exact XCDM model. Results therein show that direct fitting to the $X C D M$ model provides smaller errors than the cosmographic approaches, so the direct fitting allows us to easily spot differences from $\Lambda C D M$ model (for instance $j_{0} \neq 1$ ), as expected from data which don't correspond to $\Lambda$ CDM. Moreover, the order of the expansion ultimately affects the posterior constraints on the cosmographic parameters: there is some evidence of $j_{0} \neq 1$ for the $\theta_{1}$ set, which unexpectedly disappears for the $\theta_{2}$ set.
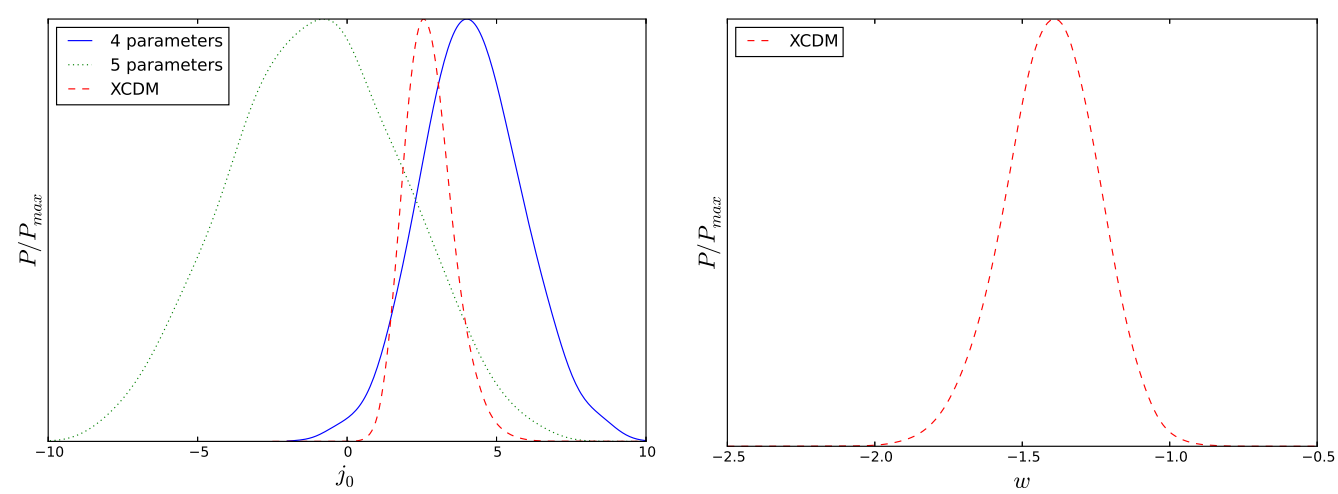

Figure 1: As shown in [11]; Left panel: Posterior probability for $j_{0}$ considering cosmographic expansions in $z$-redshift of four parameters $\left(\theta_{\mathbf{1}}\right)$, five parameters $\left(\theta_{\mathbf{2}}\right)$ and the exact $X \mathrm{CDM}$ model $\left\{\Omega_{m}=0.3, \omega_{X}=-1.3\right\}$. Right panel: Posterior probability for the dark energy equation of state parameter $\omega$ in agreement with the $\omega=-1.3$ value.

\section{Towards a better understanding of Cosmography in extended theories of gravity}

The surprising limitations described in Sec. 1 referred either to the drawbacks in the Taylor ex- 
pansions truncation or to the inability of targeting $\Lambda \mathrm{CDM}$ against simple dark energy theories, i.e., those with a minimal coupling and a constant equation of state representing the dark fluid. Nonetheless, the intense interest in the latest years on several types of gravitational theories beyond General Relativity, poses the question of the accuracy of cosmographic methods in spotting and eventually constraining such theories, or more to the point, of the intrinsic limitations that non-minimal couplings between geometry and matter and/or the presence of higher-order equations may have when such scenarios are subject to a cosmographic treatment. Most of the previous literature devoted to taking into account modern cosmography in the context of extended theories of gravity usually lacked any criticism and focused on finding constraints for classes of theories [7, 13]. Moreover the literature at hand has often assumed General Relativity represents our Universe today exactly and thus assigned strict priors to the extra parameters. More recently [11] has performed a more general treatment for both $K$-essence theories, scalar-tensor $f(R)$ theories and Galileons models, i.e., extended gravitational theories with either second order equations, higher-order equations or non-minimal couplings were addressed respectively there. Difficulties in those three classes shared the common denominator of the presence of extra parameters, a fact that prevents us from finding a one-to-one correspondence between the derivatives of the theory and the cosmographic parameters. Let's summarise some of the main results of [11] as follows,

\section{1 $K$-essence theories}

For a theory with an action - in appropriate units - given by

$$
\mathscr{S}=\int \mathrm{d}^{4} x \sqrt{-g}\left[\frac{1}{2} R-\frac{1}{2} \omega(\phi) \partial_{\mu} \phi \partial^{\mu} \phi-V(\phi)+\mathscr{L}_{m}\right],
$$

where $\mathscr{L}_{m}$ is the matter Lagrangian, $\omega(\phi)$ the factor that renormalises the scalar field $\phi$ and $V(\phi)$ the potential, the mapping between cosmographic parameters and derivatives of the potential $V$ and the factor $\omega$ when evaluated today only requires fixing one extra parameter [11]. Thus, the derivatives of the potential ${ }^{1}$ evaluated at $z=0$, can be written ${ }^{2}$ as

$$
\begin{aligned}
& \frac{V_{0}}{H_{0}^{2}}=2-q_{0}-\frac{3 \Omega_{m}}{2}, \frac{V_{z 0}}{H_{0}^{2}}=4+3 q_{0}-j_{0}-\frac{9 \Omega_{m}}{2}, \\
& \frac{V_{2 z 0}}{H_{0}^{2}}=4+8 q_{0}+j_{0}\left(4+q_{0}\right)+s_{0}-9 \Omega_{m}, \\
& \frac{V_{3 z 0}}{H_{0}^{2}}=j_{0}^{2}-l_{0}-q_{0} j_{0}\left(7+3 q_{0}\right)-s_{0}\left(7+3 q_{0}\right)-9 \Omega_{m} .
\end{aligned}
$$

The standard assumption herein in order to recover a one-to-one correspondence consists of connecting $\Omega_{m}$ and the cosmographic deceleration parameter $q_{0}$ as in the $\Lambda$ CDM model, i.e., $\Omega_{m} \approx$ $2 / 3\left(1+q_{0}\right)$. Despite this assumption, cosmography can be shown [11] to perform far worse than other model-independent approaches such as Gaussian process regression [14] for which errors are much smaller and no assumption about the model behaviour today needs be made, so any attempt at reconstructing $K$-essence theories following a cosmographic approach seems to be disfavoured with respect to Gaussian regression. Moreover, dependence in the order expansion is also present, resulting with after $z \sim 0.5$ no useful constraints for $V(\phi)$ are derived for a $\theta_{2}$ realisation, whereas

\footnotetext{
${ }^{1}$ Analogous results are obtained for the expansion of $\omega(\phi)$ derivatives evaluated today.

${ }^{2}$ Field equations in a spatially-flat FLRW were used.
} 
for the $\theta_{1}$ counterpart good constraints are obtained up to $z \sim 1$, showing the fragility of the method for these theories.

\section{2 $f(R)$ theories}

The understanding of limitations in cosmography for theories involving higher-order equations, can be exemplified by the paradigmatic $f(R)$ scalar-tensor theories which in the metric formalism give rise to fourth-order equations. Once again, a mapping between cosmographic parameters and derivatives of the gravitational Lagrangian $f(R)$ can be found [11]. The price to pay is the existence of two extra free parameters which can be thought of as the first and the second derivatives of the $f(R)$ Lagrangian evaluated today. Previous works [7] fixed those values so that the $f(R)$ Lagrangian was forced to coincide with General Relativity at $z=0$, namely $f_{R}(z=0)=1$ and $f_{R R}(z=0)=0$. However such naive priors may lead to either singularities or instabilities occuring [?], apart from the fact that cosmological values for $f(R)$ derivatives today may be different from GR exact values and still produce viable cosmological models [15]. Thus one is led to abandon the one-to-one correspondence between the $f(R)$-derivatives and the cosmographic parameters, so either priors over these $f(R)$ parameters or alternative tests are required [16]. Whenever either those priors are too strict or marginalisation of extra parameters is not carefully performed, the obtained constraints can lead to a reconstruction of the $f(R)$ models which are not capable of generating the SNIa mock data used to test the method. A combination a wide set of priors through extensive Markov Monte Carlo Chain simulations is performed in [17] and is expected to improve the predictability of the method also making use of other astrophysical probes, such as Baryon Acoustic Oscillations and $H(z)$ measurements.

\subsection{Galileons theories}

Finally, we could exemply the cosmography limitations when dealing with theories which have higher-order derivatives but this time in the matter sector, such as Galileons [18]. Thus for one of the simplest Galileon models involving three coupling constants, analogous issues as those described for $f(R)$ theories show up, due to the existence of two additional free parameters. As remarked in [11], the sole advantage of cosmographic treatment for Galileons theories is that since the gravitational sector does not involve higher-order derivatives the errors are not as large as those obtained in the $f(R)$ gravity case. This could also be understood by the fact that the expansion of the scalar field for Galileons up to second order only depends on $q_{0}$ and not on higher cosmographic parameters [11].

\section{Conclusions and Prospects}

At the present stage, the cosmographic approach seems to be plagued with drawbacks, previously overlooked in the literature, such as the dependence of results with the chosen independent variable in which the cosmographic expansion is performed as well as the lack of hints of the required expansion order which minimises the induced errors. These two facts can for instance lead to not correctly spotting fiducial models which slightly differ from the Concordance cosmological $\Lambda \mathrm{CDM}$ model. 
Moreover, the reconstruction process based on cosmography for theories containing extra degrees of freedom, such as quintessence-like theories, $f(R)$ gravities or Galileons and, by extension other modified theories, seem to lead to completely unconstrained parameters of the models under consideration when either $a$ ) Einsteinian gravity is assumed as a benchmark for models' behaviour today, or $b$ ) narrow priors or limited marginalisations for extra parameters are considered. Such cosmographic treatment may prevent us from ruling models which exhibit very unrealistic cosmological background evolutions and more dangerously, could lead to think of the viable character of models that are already excluded thanks to the use of other methods.

Consequently, it might seem that the cosmographic approach - as traditionally thought - is a strongly limited tool for theory reconstruction. In fact other limitations, apart from those explained in the bulk of this communication might appear, namely there are at least two effects which, for the sake of simplicity, were neglected in this communication. The first one considers the eventual role of the spatial curvature $\Omega_{k}$ in fixing constraints, due to the fact that $\Omega_{k} \neq 0$ can induce a time variation for the dark energy equation of state [19] and eventually distort the cosmological constraints as recently claimed in [20]. The second, additional limitations concerns other effects such as both gravitational and Doppler lensing [21], or even local gravitational redshifts [22], which may involve the appearance of extra scatters in the Hubble diagram, a fact which might degrade cosmological constraints as obtained from SNe Ia data analysis. Although quantified and included as an extra error SNe Ia analysis within the paradigm of the $\Lambda \mathrm{CDM}$ model [12], the impact that such scatters may have in theories of modified gravity, still requires a thorough study to be able to determine for which extended theories the cosmographic approach is expected not to be useful in constraining parameters.

But not everything is negative: as possible routes which deserve further exploration with the hope of curing limitations in Cosmography we could mention:

1. the need for a clear definition of the adequate auxiliary variable(s), together with the comprehension of their range of validity and extensive testing against mock data from several catalogues. In this respect, the determination of a convenient pivot redshift, around which the performed expansions become optimal could shed light on whether or not the lack of competitiveness of the cosmographic method remains. Clearly, such a pivot choice is expected to severely depend upon the redshift distribution of the considered astrophysical catalogue. In other words, this adaptive study may help us to understand how the redshift distribution of different samples influences the results of some ongoing and future surveys, such as DES and LSST.

2. a robust statistical method involving, for instance, full freedom in the priors and a combined analysis of several probes which could in principle establish a correlation between the number of data points, location in the redshift space and the optimal number of cosmographic parameters, as well as Bayesian evidence enabling us to provide some criteria to rule out regions in the parameter space of classes of models.

3. Finally, as mentioned in the bulk of the communication, the use of well posed priors over the extra parameters in higher-order theories which allow us to obtain competitive constraints for such theories, even when lensing and local effects are neglected. Work in progress in [17] is 
in this direction for several classes of theories, namely scalar-tensor and extended theories of torsion.

Finally, the aim of the present communication has been to illustrate the limitations of Cosmography by making sole use of one observable, namely supernovae of type Ia, with the aim of diverting from the technicalities of a multi-observable treatment, which might have obscured the origin of our results. Although mainly in the context of Einstein gravity different observables have been used to constrain cosmographic parameters, such as $H(z)$ data, baryon acoustic oscillations, gamma-ray bursts, angular distances to galaxy clusters [23], it is easier to understand both the cause and the extent of possible limitations with only one observable. We shall present a combined probes analyses, namely $\mathrm{H}(\mathrm{z}), \mathrm{BAO}$ and extend the supernovae treatment in our future work [17].

\section{Acknowledgments}

The author acknowledges financial support from the University of Cape Town (UCT) Launching Grants programme, National Research Foundation grant 99077 2016-2018, Ref. No. CSUR 150628121624, MINECO (Spain) projects FIS2014-52837-P, FPA2014-53375-C2-1-P, CSIC ILINK1019 and Consolider-Ingenio MULTIDARK CSD2009-00064. He would also like to thank the Yukawa Institute for Theoretical Physics (Kyoto, Japan) for their hospitality and the invitation to present these results.

\section{References}

[1] E. J. Copeland, M. Sami and S. Tsujikawa, Int. J. Mod. Phys. D 15, 1753 (2006) [hep-th/0603057]; D. H. Weinberg, M. J. Mortonson, D. J. Eisenstein, C. Hirata, A. G. Riess and E. Rozo, Phys. Rept. 530, 87 (2013) [arXiv:1201.2434 [astro-ph.CO]]; S. Nojiri and S. D. Odintsov, Phys. Rept. 505, 59 (2011) [arXiv:1011.0544 [gr-qc]]; S. Capozziello and V. Faraoni, Beyond Einstein Gravity, Springer, 2010; L. Amendola and S. Tsujikawa, Dark Energy, Theory and Observations, Cambridge University Press 2015. A. Joyce, L. Lombriser and F. Schmidt, arXiv:1601.06133 [astro-ph.CO].

[2] S. Weinberg Gravitation and Cosmology: Principles and Applications of the General Theory of Relativity (Wiley, New York, 1972) ; E. R. Harrison, Nature, 260, 591 (1976).

[3] G. M. Bernstein and B. Jain, Astrophys. J. 600, 17 (2004) [astro-ph/0309332]; M. Visser, Gen. Rel. Grav. 37, 1541 (2005) [gr-qc/0411131]; M. Bouhmadi-Lopez, S. Capozziello and V. F. Cardone, Phys. Rev. D 82, 103526 (2010) [arXiv:1010.1547 [gr-qc]].

[4] C. Cattoen and M. Visser, Class. Quant. Grav. 24, 5985 (2007).

[5] A. Aviles, C. Gruber, O. Luongo and H. Quevedo, Phys. Rev. D 86, 123516 (2012) [arXiv:1204.2007].

[6] K. Bamba, S. Capozziello, S. Nojiri and S. D. Odintsov, Astrophys. Space Sci. 342, 155 (2012) [arXiv:1205.3421 [gr-qc]].

[7] A. Aviles, A. Bravetti, S. Capozziello and O. Luongo, Phys. Rev. D 87, no. 4, 044012 (2013) [arXiv:1210.5149 [gr-qc]]. ; Phys. Rev. D 87, no. 6, 064025 (2013)

[8] S. Capozziello, R. Lazkoz and V. Salzano, Phys. Rev. D 84 (2011) 124061 [arXiv:1104.3096 [astro-ph.CO]]. 
[9] S. Weinberg Gravitation and Cosmology: Principles and Applications of the General Theory of Relativity (Wiley, New York, 1972) ; E. R. Harrison, Nature, 260, 591 (1976).

[10] P. K. S. Dunsby and O. Luongo, arXiv:1511.06532 [gr-qc].

[11] V. C. Busti, A. de la Cruz-Dombriz, P. K. S. Dunsby and D. Sáez-Gómez, Phys. Rev. D 92, no. 12 , 123512 (2015) [arXiv:1505.05503 [astro-ph.CO]].

[12] N. Suzuki et al., Astrophys. J. 746, 85 (2012) [arXiv:1105.3470].

[13] A. Aviles, A. Bravetti, S. Capozziello and O. Luongo, [arXiv:1302.4871 [gr-qc]].

[14] R. Nair, S. Jhingan, and D. Jain, J. Cosmol. Astropart. Phys. 01 (2014) 005 [arXiv:1306.0606].

[15] Á. de la Cruz-Dombriz, P. K. S. Dunsby, S. Kandhai and D. Sáez-Gómez, Phys. Rev. D 93 (2016) no.8, 084016 [arXiv:1511.00102 [gr-qc]].

[16] F. D. Albareti, J. A. R. Cembranos, A. de la Cruz-Dombriz and A. Dobado, JCAP 1307 (2013) 009; [arXiv:1212.4781 [gr-qc]]; A. de la Cruz-Dombriz, P. K. S. Dunsby, V. C. Busti and S. Kandhai, Phys. Rev. D 89 (2014) 6, 064029 [arXiv:1312.2022 [gr-qc]]; B. Jain, V. Vikram and J. Sakstein, Astrophys. J. 779 (2013) 39 [arXiv:1204.6044 [astro-ph.CO]]; L. Lombriser, A. Slosar, U. Seljak and W. Hu, Phys. Rev. D 85, 124038 (2012) [arXiv:1003.3009 [astro-ph.CO]]; L. Lombriser, F. Schmidt, T. Baldauf, R. Mandelbaum, U. Seljak and R. E. Smith, Phys. Rev. D 85, 102001 (2012) [arXiv:1111.2020 [astro-ph.CO]]; Y. -S. Song, H. Peiris and W. Hu, Phys. Rev. D 76, 063517 (2007) [arXiv:0706.2399 [astro-ph]]; Y. -S. Song, W. Hu and I. Sawicki, Phys. Rev. D 75, 044004 (2007) [astro-ph/0610532]. M. A. Resco, A. de la Cruz-Dombriz, F. J. L. Estrada and V. Z. Castrillo, arXiv:1602.03880 [gr-qc]. A. de la Cruz-Dombriz, E. Elizalde, S. D. Odintsov and D. Saez-Gomez, arXiv:1603.05537 [gr-qc].

[17] Á. de la Cruz-Dombriz, P. K. S. Dunsby, O. Luongo and L. Reverberi, work in progress.

[18] C. Deffayet, S. Deser and G. Esposito-Farese, Phys. Rev. D 80, 064015 (2009) [arXiv:0906.1967 [gr-qc]].

[19] C. Clarkson, M. Cortês, and B. Bassett, J. Cosmol. Astropart. Phys. 08 (2007) 011 [arXiv:astro-ph/0702670].

[20] C. D. Leonard, P. Bull and R. Allison, arXiv:1604.01410 [astro-ph.CO].

[21] D. E. Holz and R. M. Wald, Phys. Rev. D 58, 063501 (1998) [arXiv:astro-ph/9708036]; L. Hui and P. B. Greene, Phys. Rev. D 73, 123526 (2005) [arXiv:astro-ph/0512159]; D. J. Bacon, S. Andrianomena, C. Clarkson, K. Bolejko, and R. Maartens, Mon. Not. R. Astron. Soc. 443, 1900 (2014) [arXiv:1401.3694].

[22] R. Wojtak, T. M. Davis, and J. Wiis, [arxiv:1504.00718].

[23] V. Vitagliano, J.-Q. Xia, S. Liberati, and M. Viel, J. Cosmol. Astropart. Phys. 03 (2010) 005 [arXiv:0911.1249]; L. Xu and Y. Wang, Phys. Lett. B 702, 114 (2011) [arXiv:1009.0963]; M. Demianski, E. Piedipalumbo, C. Rubano, and P. Scudellaro, Mon. Not. R. Astron. Soc. 426, 1396 (2012) [arXiv:1206.7046]; R. F. L. Holanda, J. S. Alcaniz, and J. C. Carvalho, J. Cosmol. Astropart. Phys. 06 (2013) 033 [arXiv:1303.3307]. 